\title{
Patients for Patient SAFety
}

\section{SeeteshGhose *}

\section{BACKGROUND}

Patient safety is the absence of preventable harm to a patient during the process of health care. The discipline of patient safety is the coordinated efforts to prevent harm, caused by the process of health care itself, from occurring to patients. The frequency and magnitude of avoidable adverse patient events was not well known until the 1990s, when multiple countries reported staggering numbers of patients harmed and killed by medical errors. Some of the most common and worrisome patient issues are wrong site surgery, medication errors, healthcare acquired infection, falls , readmissions and diagnostic error.Recognizing that healthcare errors impact 1 in every 10 patients around the world, the World Health Organization calls patient safety an endemic concern. Although human factors,system factors and equipment/infrastructure factors were considered issues in patient safety, patient as a stakeholder for his own safety was realized as late as1999. In October 2004 WHO launched World Alliance for Patient Safety in response to a World Health Assembly Resolution (2002) urging WHO and Member States to pay the closest possible attention to the problem of patient safety. Its establishment underlined the importance of patient safety as a global health-care issue. The goal was to develop standards for patient safety and assist UN member states to improve the safety of health care. The Alliance raises awareness and political commitment to improve the safety of care and facilitates the development of patient safety policy and practice in all WHO Member States.

\section{Why "Patients for Patient SAFety"}

- Patientsandtheirattendantsseethingsthatbusyhealth-careprovidersoftendonot.

- Offertherichestresourceofinformationrelatedtomedicalerrorsasmanyofthemhavewitnessedeverydetailofsystemsf ailuresfromthebeginningtilltheend.

- Patientshavemuchmoretoofferthanmerereminderstohealth-careproviders, administratorsandpolicymakersthatw earevictimsoftragicmedicalerrors.

- Thevoiceofpatientsandfamilieswhohavesufferedpreventablemedicalinjuryisapowerfulmotivationalforcefor health careproviderswhowish,first,todonoharm

- Whenpatientsregisterconcerns, theyareoften perceivedasadversarialthreatsthatlackevidence,ratherthan potential knowledgecontributions.

- Patientsandtheirfamilieshavegenuineadditionalneedsandwantswhenthingsactuallygowrong.

- "PatientsforPatientSafety"emphasizesthecentralrolepatientsandconsumerscanplayineffortstoimprovethequalitya ndsafetyofhealthcare.

\section{How to achieve"Patients for Patient Safety"}

\section{Education and Awareness}

Actions that need to be taken in individual hospitals and health systems include:

- Establishing interactive, interdisciplinary education programs that bringtogether patients and professionals by targeting:

a. The general public, including patients, families, media

Explaining about

- Definition and principles of patient safety

- Frequency of medical error

- How to safeguard your own care and partner with your providers

- What to do if you experience a mistake or error

b. Healthcare organizations and professionals

Explaining about

- Patient/family perspective is important and should be activelyintegrated into culture of institution.

c. The behavioural health community, including counsellors andsocial workers

\footnotetext{
* Dr.SeeteshGhose,MD, Professor\& Head, Department of Obstetrics and Gynaecology,

MGMCRI,Pondicherry,
} 
Explaining about

- Experience of medical error differs from other types of trauma — patients and families who experience harm due to a medical errormay need specific types of support and advocacy.

\section{Building A Culture of Patient and Family-Centered Patient Safety}

Meaningful change cannot take place without a fundamental change inthe culture of patient safety. The following actions are aimed at buildingpartnerships with patients and families.

Actions that need to be taken by individual hospitals and organizations:

- Teach and encourage effective communication skills for patients, theirfamilies and healthcare professionals

- Train and utilize patient representatives for patient safety advocacy inhospitals and health systems

- Implement Patient and Family Advisory Councils in each hospital andhealthcare organization

- Incorporate patient and family representation on Boards of Trustees

- Develop patient safety task forces and/or coalitions in each state

\section{Research}

Suggested areas of internal and external research:

- "Bridging the Gap": Effective methods for building relationships andcommunication between patients, caregivers, and providers.

- Disclosure - Methods and their effects on patients and families.

- Short- and long-term effects of integrating patients and families into thehealthcare system.

- Review of current patient safety information and resources available forpatients and families, and their effectiveness.

- Post-traumatic stress specific to medical error.

- Team relationships (including patients and families)

\section{Support Services}

There are three phases of medical error: preventing the error, preventingharm caused by the error, and mitigating the effects of a harmful error.

Support services are needed to address this last category.

$\otimes$ A national resource center and information line

A peer resource counselling program to connect patients who haveexperienced a medical error with trained individuals who have alreadybeen through the experience

\ National training programs

\ Individual organizations and local coalitions should provide:

\ Support groups

$\nabla$ Disclosure and communications programs

$\bigotimes$ A long-term goal in this area is:Emergency line

\section{Organizations associated With “Patients for Patient Safety"}

\ Governmental organizations: - WHO [World Alliance for Patient Safety, Patients for Patient Safety (PfPS)], Aus and NZ [Therapeutic Goods Administration and Adverse Drug Reactions Advisory Committee, Australian Commission on Safety and Quality in Health Care, New Zealand Health Quality \& Safety Commission],UK[National Patient Safety Agency, National Institute for Health and Clinical Excellence],USA[ Composition, Agency for Healthcare Research and Quality, Food and DrugAdministration].

\ Independent organizations:-Aus (Australian Patient Safety Foundation), Canada (Canadian Patient Safety Institute, Institute for Safe Medication Practices Canada)

Germany(German Agency for Quality in Medicine, German Coalition for Patient Safety),UK (The Health Foundation),USA (American Society of Medication Safety Officers, National Quality ForumLeapfrog, Joint Commission on Accreditation of Healthcare Organizations, Pittsburgh Regional Health Initiative, National Patient Safety Foundation, United States Pharmacopeia, Institute for Safe Medication Practices ,Safe Care Campaign, TMIT, ECRI Institute, Institute for Safety in Office-Based Surgery,Clarity Group, Inc.) 


\section{FUNCTIONS}

Patient safety organizations may use several approaches to reduce adverse events

$>$ Collect data on the prevalence and individual details of errors.

$>$ Analyze sources of error by root cause analysis.

$>$ Propose and disseminate methods for error prevention.

$>$ Design and conduct pilot projects to study safety initiatives, including monitoring of results.

$>$ Raise awareness and inform the public, health professionals, providers, purchasers and employers.

$>$ Conduct fundraising and provide funding for research and safety projects

$>$ Advocate for regulatory and legislative changes

\section{CONCLUSION}

Over the past ten years, patient safety has been increasingly recognized as an issue of global importance, but much work remains to be done. Patients' perceptions with regard to Patient Safety have to be addressed.T he patients should be involved in making the hospitals safer places for them. There should be more opportunitiesofinteractionbetweenservic eproviders,patients,families,administratorsand policymakers. Systemshouldbedesignedwhichaddressgrievances/doubts/ suggestions.Awarenesslevelsaboutpatientsafety tobeenhancedbyprovidinginformationaboutpatientsafetyinthehealthorg anizationssuchthatitreachesthecommonman. 
